\title{
ASYMPTOTICALLY UNBIASED INFERENCE FOR ISING MODELS
}

\author{
H. KÜNSCH, ${ }^{*}$ University of Tokyo
}

In his recent paper on statistical inference for binary Markov fields Pickard (1982) writes that 'maximum likelihood inference for the interaction parameter cannot be performed for large sized lattices'. The reason for this is that the solution of the asymptotic normal equations has a bias which is of the same or higher order as the random fluctuations. We should like to show here that this bias can be removed by a simple modification of the normal equations. The same modification was proposed by Guyon (1982) in the case of Gaussian random fields; it consists essentially in replacing biased sample product moments by unbiased ones. We take as our estimator the solution of the equations

$$
D H(a)_{\nu}=\alpha_{\nu} /\left|\mathscr{C}_{\nu}\right| Q_{\nu}(\boldsymbol{x}) \quad(\nu=0, \cdots, 1) .
$$

On p. 351 Pickard discusses the version with $N^{-1}$ instead of $\alpha_{\nu} /\left|\mathscr{C}_{\nu}\right|$. The difference between $\alpha_{\nu}$ and $\left|\mathscr{C}_{\nu}\right| / N$ is of the order $O\left(N^{-1 / d}\right)$.

If no phase transition occurs, then we have by general results from statistical mechanics that

$$
D H(a)_{\nu}=\alpha_{\nu} \mathscr{E}_{\infty}\left(\prod_{i \in \theta} x_{i}\right)
$$

where $\theta$ is any clan of the equivalence class $\mathscr{C}_{\nu}$ and $\mathscr{E}_{\infty}$ denotes the expectation with respect to the infinite Gibbs distribution $P_{\infty}$. Because the number of terms in $Q_{\nu}(x)$ is just $\left|\mathscr{C}_{\nu}\right|$, we obtain from (1) and (2)

$$
D H(\hat{a})_{\nu}-D H(a)_{\nu}=\alpha_{\nu} N /\left|\mathscr{C}_{\nu}\right| . N^{-1}\left(Q_{\nu}(\boldsymbol{x})-\mathscr{C}_{\infty}\left(Q_{\nu}(\boldsymbol{x})\right) .\right.
$$

where $\hat{\boldsymbol{a}}$ is the solution of (1).

If we are sufficiently far away from phase transition, then under $\boldsymbol{P}_{\infty} N^{-\frac{1}{2}}(\boldsymbol{Q}(\boldsymbol{x})-$ $\left.\mathscr{C}_{\infty}(\boldsymbol{Q}(\boldsymbol{x}))\right) \stackrel{\mathfrak{M}}{\rightarrow} \mathbf{N}\left(\mathbf{0}, \boldsymbol{D}^{2} H\right)$, see e.g. Dobrushin and Tirozzi (1977), Section 1.3, or Künsch (1982), Section 4. Clearly this implies that under $P_{\infty} N^{\frac{1}{2}}(\hat{a}-a) \stackrel{M}{\rightarrow} \mathbf{N}\left(\mathbf{0},\left(\boldsymbol{D}^{2} H\right)^{-1}\right)$.

What happens if we replace $P_{\infty}$ by the sequence of laws $P_{\Lambda}$ ? From (3) we obtain

$$
\begin{gathered}
D H(\hat{\boldsymbol{a}})_{\nu}-D H(\boldsymbol{a})_{\nu}=\alpha_{\nu} N /\left|\mathscr{C}_{\nu}\right| \cdot N^{-1}\left(Q_{\nu}(\boldsymbol{x})-\mathscr{C}_{\Lambda}\left(Q_{\nu}(\boldsymbol{x})\right)\right. \\
+\alpha_{\nu} /\left|\mathscr{C}_{\nu}\right| \sum_{\theta \in \mathscr{C}_{\nu}}\left(\mathscr{C}_{\Lambda}\left(\prod_{i \in \theta} x_{i}\right)-\mathscr{C}_{\infty}\left(\prod_{i \in \theta} x_{i}\right)\right) .
\end{gathered}
$$

The asymptotic normality of the first term follows from Theorem 1 of Pickard. In order to estimate the second term, we need to know how fast $\mathscr{E}_{\Lambda}\left(\prod_{i \in \theta} x_{i}\right)$ converges to $\mathscr{E}_{\infty}\left(\prod_{i \in \theta} x_{i}\right)$ for a fixed clan $\theta$. But at least if the interaction is weak enough, it follows

Received 20 April 1983.

* Present address: Mathematische Statistik, ETH Zentrum, CH-8092 Zürich, Switzerland. 
from Corollary 2.4 of Künsch (1982) that this convergence is exponential, i.e.

$$
\left|\mathscr{E}_{\Lambda}\left(\prod_{i \in \theta} x_{i}\right)-\mathscr{C}_{\infty}\left(\prod_{i \in \theta} x_{i}\right)\right| \leqq C_{1} \exp \left(-C_{2} d\left(\theta, \Lambda^{c}\right)\right)
$$

where $d\left(\theta, \Lambda^{c}\right)$ denotes the distance between $\theta$ and the region outside $\Lambda$. From (5) it follows that the second term in (4) is of the order $O\left(N^{-1}\right)$ and therefore the asymptotic behaviour of $\hat{a}$ is the same under $\boldsymbol{P}_{\Lambda}$ as under $\boldsymbol{P}_{\infty}$.

It is now clear that we can construct confidence ellipsoids and acceptance regions at an asymptotically correct level, provided $\mathrm{DH}$ and $\mathrm{D}^{2} \mathrm{H}$ are known and we use the solution of the equations (1).

\section{References}

Dobrushin, R. L. AND Tirozzi, B. (1977) The central limit theorem and the problem of equivalence of ensembles. Commun. Math. Phys. 54, 173-192.

GuYON, X. (1982) Parameter estimation for a stationary process on a $d$-dimensional lattice. Biometrika 69, 95-105.

KüNSCH, H. (1982) Decay of correlations under Dobrushin's uniqueness condition and its applications. Commun. Math. Phys. 84, 207-222.

PiCKARD, D. (1982) Inference for general Ising models. J. Appl. Prob. 19 A, 345-357. 